\title{
Concentración de cadmio en marcas de cigarrillos de tabaco comercializados en Guayaquil, Ecuador
}

\author{
Concentration of cadmium in tobacco cigarette brands traded in \\ Guayaquil, Ecuador
}

\section{Connie Jacqueline Durán Barros ${ }^{*}$ \& Rubén Castro}

\author{
${ }^{1}$ Consultora Independiente, Instituto Nacional de Pesca. \\ ${ }^{2}$ Docente investigador de la Facultad de Ciencias Naturales, Universidad de Guayaquil, \\ Av. Raúl Gómez Lince s/n y Av. Juan Tanca Marengo, Guayaquil, Ecuador.
}

Recibido de abril 2020; recibido en forma revisada 26 de mayo 2020, aceptado 1 de junio 2020 Disponible en línea 26 de junio 2020

\section{Resumen}

El cadmio es un metal pesado tóxico cuyo tiempo de vida en el organismo es de aproximadamente 30 años, la exposición al mismo ha sido relacionada con distintos tipos de cáncer. El objetivo del presente estudio fue analizar la existencia de contaminación por cadmio en cigarrillos de tabaco que se expenden en Guayaquil. Se recolectaron un total de 22 muestras, éstas fueron clasificadas por marca y país de origen. El contenido de cadmio se determinó mediante la técnica de espectrofotometría de absorción atómica de horno de grafito, encontrándose en efecto, un promedio de $1,89 \pm 1,75 \mathrm{mg} / \mathrm{kg}$. Existen tres grupos de cigarrillos con diferencias significativas en cuanto a la concentración de cadmio (Anova; $p<0,005$ ), siendo la marca "COS", de procedencia china, cuyo expendio es de manera ilícita, la que mayor concentración presentó $(4,73 \pm 1,14 \mathrm{mg} / \mathrm{kg})$. Los cigarrillos manufacturados en el Ecuador ocupan el segundo lugar en lo que respecta a concentraciones de cadmio $(1,49 \pm 0,58 \mathrm{mg} / \mathrm{kg})$, llamando la atención los cigarrillos de procedencia india, también de expendio ilícito, los que obtuvieron la menor concentración $(0,418 \pm 0,25 \mathrm{mg} / \mathrm{kg})$. Se planteó una propuesta para reducir la incidencia de $\mathrm{Cd}$ en el proceso de producción de cigarrillos de tabaco de producción nacional.

Palabras Claves: cadmio, cigarrillos, contaminación, cáncer, Ecuador, Guayaquil.

\begin{abstract}
Cadmium is a toxic heavy metal whose life in the human body is of approximately 30 years, the exposure to it has been related to different types of cancer. The aim of this study was to analyze the presence and concentration of cadmium in tobacco cigarettes that are sold in Guayaquil. A total of 22 samples were collected and grouped by brand and country of origin. Cadmium content was determined using the graphite furnace atomic absorption spectrophotometry technique, with an average of $1.89 \pm 1.75 \mathrm{mg} / \mathrm{kg}$. There are three groupsof cigarettes with significant differences in terms of cadmium concentration (Anova; $\mathrm{p}<0.005$ ), being the brand "COS" made in China, whose sale is illegal, the one with the highest Cd concentration $(4.73 \pm 1.14 \mathrm{mg} / \mathrm{kg})$. Cigarettes manufactured in Ecuador occupy thesecond place in terms of concentrations of cadmium (1.49 \pm $0.58 \mathrm{mg} / \mathrm{kg}$ ), attracting attention the cigarettes of Indian origin, also of illegal sale, which obtained the lowest concentration $(0.418 \pm 0.25 \mathrm{mg} / \mathrm{kg})$. A proposal was made to reduce the incidence of cadmium in the production process of tobacco cigarettes production at national level.
\end{abstract}

Keywords: cancer cadmium, cigarettes, Ecuador, Guayaquil, pollution, tobacco.

\section{Introducción}

La contaminación ambiental es uno de los problemas más importantes que enfrenta el planeta, esta surge cuando se produce un desequilibrio debido a la adición de cualquier sustancia ajena al ambiente en una cantidad tal, que cause efectos perjudiciales en los seres vivos y o materiales expuestos a dosis que sobrepasan los niveles aceptables en la naturaleza (Instituto de Salud Pública de Chile,2019).
La contaminación del suelo es definida como la acumulación de compuestos tóxicos persistentes en los suelos, productos químicos, materiales radiactivos, sales o agentes que causan enfermedades que tienen efectos adversos sobre la salud animal y el crecimiento de las plantas (Okrent, 1999).

Las causas de esta contaminación suelen ser difíciles de identificar, sin embargo, se conoce que casi todas ellas están relacionadas con la actividad antropogénica,

* Correspondencia del autor:

E-mail: ruben.castror@ug.edu.ec 
Rev. Cient. Cien. Nat. Ambien. 14(1):127-136 Junio 2020
ISSN: $2773-7772$
Durán \& Castro • Concentración de cadmio en marcas de cigarrillos de tabaco comercializados en Guayaquil, Ecuador entre estas: filtración de un vertedero, descarga de residuos industriales en el suelo, filtración de agua contaminada en el suelo, ruptura de tanques de almacenamiento subterráneos, filtración de residuos sólidos, y aplicación excesiva de pesticidas, herbicidas o fertilizantes, que por lo general contienen metales pesados (Ashraf et al., 2014).

Los metales pesados son elementos químicos que se encuentran de forma natural en la corteza terrestre, aunque en muy bajas concentraciones, poseen un alto peso molecular, masa y su densidad es alrededor de cinco veces superior en comparación con la del agua. El problema con estos elementos es que llegan a ser tóxicos incluso en concentraciones bajas. Los metales pesados tóxicos más conocidos son el arsénico, cadmio, cobalto, plomo, níquel, cromo, cobre, mercurio, estaño y zinc (Londoño et al., 2016). Una vez liberados al ambiente, mediante innumerables variedades de productos químicos, se introducen en los organismos por medio de la ingesta de alimentos, la inhalación y la capacidad de absorción de la piel y son retenidos más rápido de lo que el cuerpo puede eliminarlos, obteniendo como consecuencia una acumulación gradual en los tejidos del cuerpo (Sardar et al., 2013).

Las fuentes principales de contaminación por Cd son: la metalurgia del acero y del hierro, la minerometalurgia de metales no ferrosos, la combustión de gasolina y aceite, fabricación y aplicación de fertilizantes fosfatados, la incineración de madera, carbón o plástico, y las aplicaciones industriales de cadmio. La persistencia del cadmio en suelos es de hasta trescientos años y el $90 \%$ persiste sin transformarse. Este metal llega a los suelos de los terrenos agrícolas por medio de: deposición aérea (41\%), fertilizantes fosfatados (54\%) y por aplicación de abono de estiércol (5\%). Se ha encontrado que, en suelos contaminados, los niveles de cadmio logran alcanzar valores de hasta $1 \mathrm{mg}$ de $\mathrm{Cd}$ por gramo y el agua de ríos contaminados podría contener hasta 0,14 mg de Cd/L (Ramírez, 2002).

La población se encuentra expuesta al cadmio por diversas vías como por ejemplo la oral, por medio de la ingesta de agua y de comida contaminada con este metal (granos, frutas, hojas de vegetales, cereales, vísceras animales y pescado) (Nava \& Méndez, 2011). En algunos países del continente europeo y Norte América la ingesta diaria de cadmio oscila entre 0,01 y 0,04 mg/día (Reyes et al., 2016).

Otra forma de exposición, muy particular de los seres humanos, es el cigarrillo, el nivel de cadmio está relacionado al suelo donde se cultiva el tabaco. Ha sido probado que las plantas de tabaco poseen la capacidad específica e inusualmente alta de absorber el Cd del suelo y acumularlo en sus raíces y hojas. Esta capacidad es significativamente distinta entre las diferentes variedades de tabaco, siendo la especie Nicotiana tabacum la que acumula más $\mathrm{Cd}$ en sus hojas y raíces (Petit de Peña et al., 2014). Al fumar, la toxicidad se produce por inhalación. Los pulmones humanos son capaces de reabsorber entre el 40 y el $60 \%$ del cadmio presente en el humo del tabaco y, por consiguiente, los fumadores tienen concentraciones de cadmio en la sangre hasta cuatro veces más altas que las de los no fumadores (Chakraborty et al., 2013).

Al fumar, la inhalación es una vía de ingreso del cadmio al organismo cuya importancia es principalmente local. Las plantas de tabaco tienden a acumular cadmio en las hojas y los cultivos de tabaco son frecuentemente objeto de una abundante fertilización fosfática, que puede producir un incremento en los niveles de cadmio en los suelos (Herrera, 2000).

Los cigarrillos de tabaco, además de ser tóxicos, son potencialmente adictivos, y eso es debido a que producen liberación de dopamina en las regiones del cerebro que controlan las sensaciones de placer y bienestar; cabe recalcar que su componente, la nicotina, crea tolerancia (Leshner, 2001).

En cuanto a la distribución de $\mathrm{Cd}$ en plantas se conoce que los niveles de este metal decrecen en el orden: raíces>hojas>fruto>semilla (Davis, 1984); sin embargo, las concentraciones en la planta de tabaco son mayores en sus hojas que en sus demás órganos (Yoshihara et al., 2013). Varios estudios han mostrado que el tabaco posee una gran capacidad de absorción de $\mathrm{Cd}$ del suelo y la acumula en grandes volúmenes en las hojas (Wagner \& Yeargan, 1986; World Health Organization, 2010). Añadiendo a lo anterior, Wagner (1993) menciona que la distribución de Cd en los diferentes órganos de la planta varía según su tipo y variedad.

Internacionalmente, se han realizado algunos estudios en donde se comparan las concentraciones de $\mathrm{Cd}$ entre varias marcas de cigarrillos. Un estudio realizado por Lugon et al. (2006) determinó las concentraciones de Cd presente en cigarrillos de 13 países. Sus hallazgos fueron los siguientes: India $(0,33 \mathrm{mg} / \mathrm{kg})$, Filipinas $(2,24 \mathrm{mg} / \mathrm{kg})$, Tailandia $(1,99 \mathrm{mg} / \mathrm{kg})$; Turquía $(0,56$ $\mathrm{mg} / \mathrm{kg})$; Albania $(1,64 \mathrm{mg} / \mathrm{kg})$; Bulgaria $(1,06 \mathrm{mg} /$ $\mathrm{kg})$; Francia $(1,46 \mathrm{mg} / \mathrm{kg})$; Grecia $(0,77 \mathrm{mg} / \mathrm{kg})$; Italia $(0,82 \mathrm{mg} / \mathrm{kg})$; Argentina $(0,65 \mathrm{mg} / \mathrm{kg})$; Brasil $(0,75$ $\mathrm{mg} / \mathrm{kg})$; Ecuador $(1,95 \mathrm{mg} / \mathrm{kg})$; USA $(0,51 \mathrm{mg} / \mathrm{kg})$.

En China, el principal país productor de cigarrillos de tabaco, investigadores como Watanabe et al. (1987) reportaron concentraciones de Cd promedio de $0,93 \mathrm{mg} / \mathrm{kg}$ para cigarrillos producidos en dicho país. Del mismo modo, reportaron concentraciones de $\mathrm{Cd}$ en cigarrillos procedentes de India, con una concentración promedio de $0,35 \mathrm{mg} / \mathrm{kg}$.

Los resultados de un estudio realizado en Venezuela por Petit de Peña et al. (2014) indican que no existe diferencia significativa entre el $\mathrm{Cd}$ detectado en cigarrillos de tabaco producidos en Venezuela versus los cigarrillos que entran a dicho país por contrabando.

Un estudio real llevado a cabo por Lugon et al. (2006) 
se propuso a comparar concentraciones de $\mathrm{Cd}$ en los 3 tipos principales de tabaco cultivado para consumo humano: curado al humo (también conocido como Virginia) $(n=359)$, Burley $(n=216)$, y Oriental $(n=$ 180). Las concentraciones promedio fueron $0,78,1,27$ y $0,99 \mathrm{mg} / \mathrm{kg}$ respectivamente.

Estos resultados indican que la concentración promedio de cadmio para tabaco tipo Burley fue significativamente mayor que para el tabaco Virginia $\mathrm{u}$ oriental, demostrando que las concentraciones de Cd varían entre los diferentes tipos de tabaco.

Varios estudios han demostrado que la concentración de cadmio en la sangre para no fumadores varía entre 0,0004 a 0,001 $\mathrm{mg} / \mathrm{L}$, mientras que en fumadores los valores se encuentran entre 0,0014 a 0,004 mg/L (Combariza, 2009). Además de los efectos adversos de este metal sobre los seres humanos, el cadmio causa graves desequilibrios en el proceso de nutrición y transporte de agua en las plantas (Singh \& Tewari, 2003). Debido a la gran capacidad de acumulación de cadmio de estas plantas se ha llegado a considerarlas como potenciales candidatos para trabajos de fitoremediación de este metal (Reyes et al., 2016).

Estudios de Pernía et al. (2016) afirman que Ecuador muestra evidencias de contaminación por cadmio en suelos agrícolas, los cuales se mencionan con sus respectivas concentraciones: cultivos de palma africana $(0,02-0,68 \mathrm{mg} / \mathrm{kg} \mathrm{Cd})$, banano $(0,09$ $0,38 \mathrm{mg} / \mathrm{kg} \mathrm{Cd})$, cultivos de café $(0,02-0,30 \mathrm{mg} / \mathrm{kg}$ (d), entre otros. También se han realizado estudios en arroz, cacao, soya, y fertilizantes encontrando contaminación por cadmio (Chávez et al., 2015; López, 2017; Muñoz, 2017).

La International Agency for Research on Cancer (IARC) ha incluido algunos agentes químicos que proceden del humo del tabaco en el "Grupo I de carcinógenos humanos": benceno, cadmio, arsénico, níquel, cromo, 2-naftilamino, clorovinil, 4 aminobifenil y Be. Cuando se usan los piretroides como insecticidas en el cultivo del tabaco, algunos de los residuos de dichos componentes podrían aparecer en el humo del cigarrillo (Smith et al., 1997; Cai et al., 2002).

En el humano, los efectos causados por la exposición al cadmio están bien documentados: es irritante y tóxico respiratorio, tóxico renal y se le ha asociado con cáncer (Ramírez, 2002). Durante los últimos años, el Ministerio de Salud Pública (MSP) ha reportado un incremento significativo en el deceso por enfermedades de cáncer; en Ecuador, esta enfermedad es la segunda causa de muerte después de las enfermedades cardiovasculares (Ministerio de Salud Pública, 2018).

Además, este mismo organismo cataloga al uso de tabaco como uno de los factores de riesgo para desarrollar distintos tipos de cáncer. Con base en esto, varios estudios enfocados en analizar concentraciones de $\mathrm{Cd}$ en alimentos se han llevado a cabo en Ecuador. Por ejemplo, se han encontrado niveles de $0,15 \mathrm{mg} /$ $\mathrm{kg}, 0,09 \mathrm{mg} / \mathrm{kg}$ y $0,35 \mathrm{mg} / \mathrm{kg}$ de $\mathrm{Cd}$ en distintas formas de soya como grano, polvo y líquido respectivamente (López, 2017). Otro estudio, por Flores et al. (2018), demostró que de 36 muestras de atún analizadas, el $66 \%$ estuvo por encima de los límites máximos permisibles de Cd según las normas NTE INEN 183 y 184.

Por consiguiente, el objetivo de este estudio es estimar la contaminación por $\mathrm{Cd}$, mediante un análisis comparativo entre las concentraciones detectadas en distintas marcas de cigarrillos de tabaco comercializados en la ciudad de Guayaquil que se consumen libremente, y de este modo ampliar la información presente acerca de este metal pesado contaminante.

\section{Metodología}

\section{Área de estudio}

La presente investigación fue realizada en la ciudad de Guayaquil, en la provincia del Guayas, ubicada en la costa de Ecuador (fig. 1). En el año 2017 el Instituto Nacional De Estadísticas y Censos (INEC), reportó que la cantidad de habitantes en Guayaquil es de $2^{`} 644.891$.

Las cajetillas de cigarrillos de diferentes marcas producidos y/o comercializadosen Guayaquil fueron adquiridas en diferentes puntos de venta de la ciudad, siendo estos: gasolineras, tiendas de abarrotes, vendedores ambulantes y un centro comercial en diferentes sectores de la ciudad, donde es posible comprar este producto,cuya venta es prohibida para menores de edad.

\section{Diseño experimental}

Se seleccionaron 5 marcas distintas de cigarrillos tomando como criterio su origen, así se consideraron 4 países distribuidos de la siguiente manera: 2 de Ecuador, 1 de China, 1 de India, y 1 de Argentina. De estas, se obtuvo un total de 22 muestras cuya codificación se detalla en la tabla 1.

Con respecto a los puntos de muestreo, se seleccionaron gasolineras, tiendas de abarrote, vendedores ambulantes y tiendas especializadas de tabaco, los cuales son lugares a los que acude la mayoría de población fumadora en Guayaquil para adquirirtabaco y cigarrillos de tabaco.

Del tabaco de procedencia argentina se obtuvieron únicamente dos muestras debido a que la presentación de este no es la clásica de forma tubular enrolada dentro de un papel especial y con filtro, sino que se expende únicamente el tabaco de manera suelta en presentación de $25 \mathrm{~g}$. Además, se comercializa en puntos de venta especializados de tabaco y productos de tabaco. La recolección de las muestras se realizó al azar y no se aplicó ninguna estrategia de muestreo estándar.

\section{Cigarrillos de producción nacional}

Para los cigarrillos de producción nacional se investigaron los tipos de tabaco para cigarrillos que se cultivan 


\section{MAPA DE ÁREA DE ESTUDIO: LOCALIZACIÓN DE PUNTOS DE MUESTREO}

\section{1:100.000}
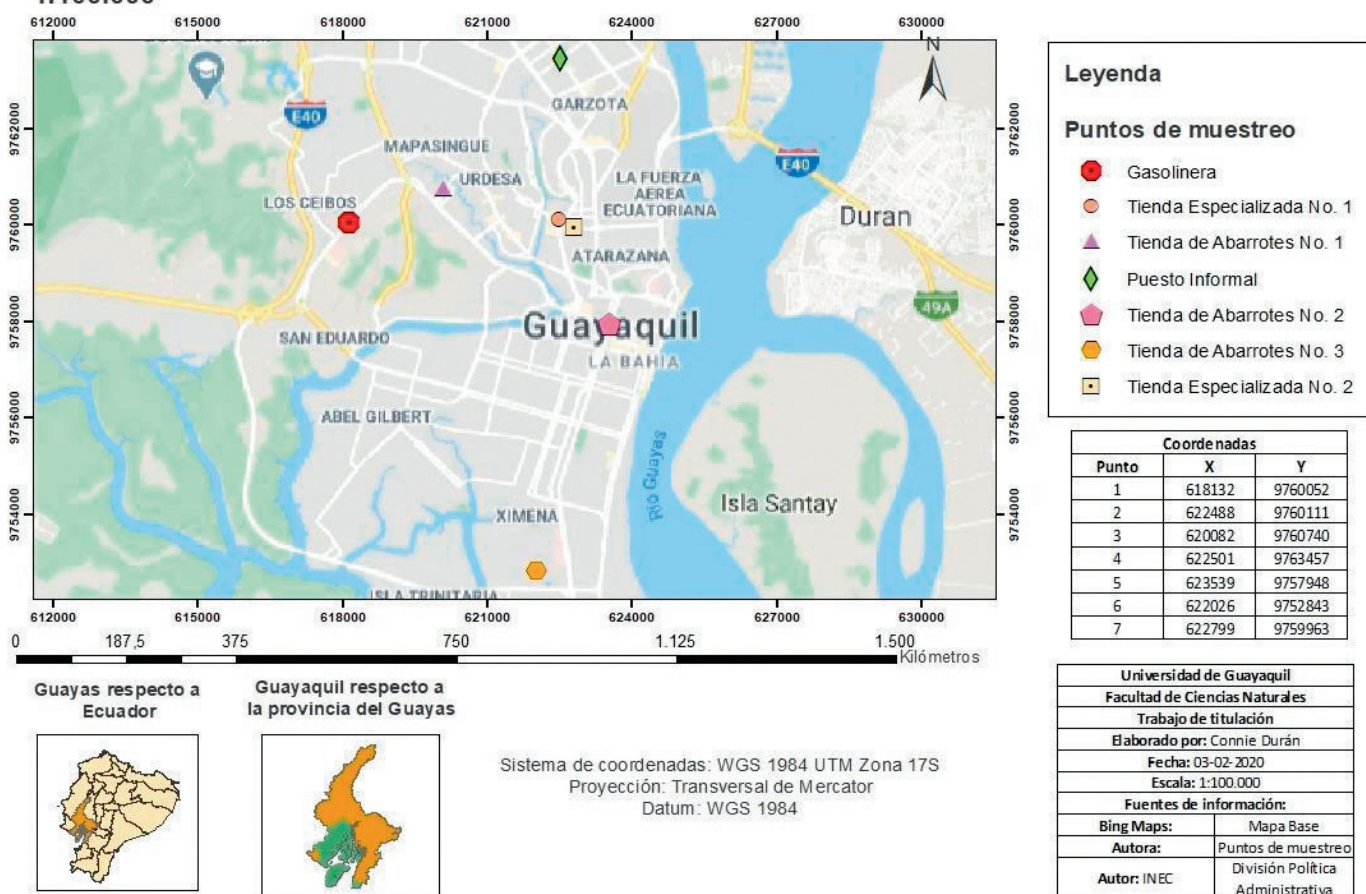

Sistema de coordenadas: WGS 1984 UTM Zona 17S Proyección: Transversal de Mercator Datum: WGS 1984

\begin{tabular}{|c|c|}
\hline \multicolumn{2}{|c|}{ Universidad de Guayaquil } \\
\hline Facultad de Ciencias Naturales \\
\hline Trabajo de titulación \\
\hline Elaborado por: Connie Durán \\
\hline Fecha: 03-02-2020 \\
\hline Escala: 1:100.000 \\
\hline Fuentes de información: \\
\hline Bing Maps: & Mapa Base \\
\hline Autora: & Puntos de muestreo \\
\hline Autor: INEC & $\begin{array}{c}\text { División Politica } \\
\text { Administrativa }\end{array}$ \\
\hline
\end{tabular}

Figura 1. Mapa de Puntos de Muestreo.

Tabla 1. Codificación y tamaño de muestras para las cinco marcas de cigarrillo de tabaco seleccionadas según su procedencia

\begin{tabular}{lll}
\hline Codificación & Origen & $\mathrm{n}$ \\
\hline NAM & Ecuador & 5 \\
NAL & Ecuador & 5 \\
COS & China & 5 \\
COU & India & 5 \\
ILS & Argentina & 2
\end{tabular}

Nota. $\mathrm{n}$ = número de muestras

y producen en Ecuador. Por su alta aceptación entre la población fumadora, se seleccionaron 2 marcas de cigarrillos de producción nacional.

En el empaque de los cigarrillos de estas marcas se pudo observar su respectivo número de lote y sello del Servicio de Rentas Internas (SRI), indicando que cuentan con los permisos respectivos para su venta, los cuales demuestran que son formalmente comercializados en el país. Al momento de su selección se optó por adquirir 5 muestras de cada marca $(n=5)$; cada muestra comprende una cajetilla de 10 unidades de cigarrillos, de los cuales sólo se tomaron aleatoriamente dos unidades por cajetilla para realizar los análisis.

\section{Cigarrillos de tabaco internacional}

Para la selección de los cigarrillos internacionales, se optó por escoger tres marcas de distintos países. Debido a su gran popularidad de consumo la primera a considerar fue una de procedencia china. Su precio de venta al público (PVP) es realmente bajo en comparación con las marcas de producción nacional, además su única presentación comercial es de 20 unidades por cajetilla. Se pudo observar que no contaban con números de lote ni tampoco sellos del SRI por lo que se asume que ingresa al país de manera ilícita. Cabe recalcar que se trata de una marca que ha ganado popularidad actualmente. El número de muestras seleccionadas fue de cinco $(n=5)$.

Otra de las marcas seleccionadas procede de India, este país ocupa el segundo lugar de los mayores productores de tabaco en el continente asiático, y tercero en el mundo luego de Brasil. Este producto, al igual que el anterior, es comercializado de forma ilícita y cuenta con las mismas características de precio, cantidad de unidades por cajetilla, y presentación.

Finalmente, se añadió una tercera marca internacional de origen argentino cuya presentación difiere de las otras marcas siendo este un paquete de 25 gramos de tabaco suelto listo para enrolar. Se encuentra disponible únicamente en tiendas especializadas de tabaco y productos de tabaco, la cual tenía un precio muy elevado en comparación a todas las demás marcas consideradas en este estudio. 


\section{Análisis de laboratorio}

Las muestras codificadas fueron llevadas al Laboratorio de Análisis Químico y Microbiológico de Alimentos de la Subsecretaría de Calidad e Inocuidad (SCI) del Ministerio de Comercio Exterior. Previo al análisis, los matraces estuvieron sumergidos durante 24 horas en solución de ácido nítrico al uno por ciento y posteriormente enjuagados con abundante agua ultra pura $(18,3 \mathrm{M} \Omega . \mathrm{cm})$. Los vasos de teflón del microondas fueron sometidos a un proceso de limpieza adicionando $10 \mathrm{~mL}$ de ácido nítrico concentrado a cada uno de ellos, sometidos al microondas a una rampa de $180^{\circ} \mathrm{C}$ durante 10 min manteniendo esta temperatura por $10 \mathrm{~min}$ adicionales, posteriormente fueron enjuagados con solución de ácido nítrico al uno por ciento y abundante agua ultra pura.

De cada cajetilla se tomaron aleatoriamente 2 cigarrillos, se descartó el filtro y se procedió a mezclar la porción del tabaco contenida en los dos cigarrillos; en el caso del tabaco de origen argentino, debido a su presentación (tabaco suelto sin papel ni filtro), solo fue necesario homogenizarlo. Posteriormente se pesó $0,30 \pm 0,05 \mathrm{~g}$ de cada una de las muestras en vasos de teflón (CEM Corporation) y se sometieron a digestión ácida en un microondas (MARSExpress, CEM) empleando $6 \mathrm{~mL}$ de ácido nítrico concentrado. Las soluciones obtenidas fueron diluidas a un volumen final de $25 \mathrm{~mL}$ con agua ultra pura, trasvasando las aguas de lavado a esta solución.

\section{Método de detección}

El contenido de Cd fue determinado mediante espectrofotometría de absorción atómica usando la técnica de horno de grafito (VARIAN modelo SpectrAA 220Z) empleando soluciones de MgNO3.6H2O y $\mathrm{NH} 4 \mathrm{H} 2 \mathrm{PO} 4$ como modificadores de matriz. El método se fundamenta en que las muestras digeridas son sometidas a un proceso térmico que consiste en las etapas de secado, carbonización, atomización y limpieza en el horno de grafito.

La espectrofotometría de absorción atómica se fundamenta en que la radiación de un elemento excitado pasa a través del vapor que contiene los átomos en estado basal de ese elemento. La intensidad de la radiación transmitida disminuye en proporción a la cantidad del elemento en estado basal en el vapor. Los átomos de metal a medir se colocan en el haz de radiación al aumentar la temperatura del horno, lo que provoca que la muestra inyectada se volatilice. Un monocromador aísla la radiación característica de la lámpara de cátodo hueco, y un dispositivo fotosensible mide la radiación atenuada transmitida (U.S. Environmental Protection Agency, 2007).

Se empleó la longitud de onda de $228,8 \mathrm{~nm}$ y los resultados fueron confirmados mediante la adición de controles de calidad como: soluciones blanco de reactivo, muestras duplicadas, muestras fortificadas y muestra certificada de referencia (IAEA 407, tejido de pescado). El límite de cuantificación (LC) del método empleado fue de $0,014 \mathrm{mg} / \mathrm{kg}$. Los valores obtenidos están expresados en $\mathrm{mg} / \mathrm{kg}$ en peso seco.

\section{Pruebas estadísticas}

Los datos obtenidos fueron tabulados en una hoja de cálculo Excel 2010 y previo al análisis estadístico se comprobaron los supuestos de la ANOVA realizando pruebas de normalidad Anderson Darling y, para identificar igualdad de varianza se empleó la prueba de Levene; seguidamente se determinó un análisis de ANOVA de una vía para comparar las medias de las concentraciones de $\mathrm{Cd}$ entre marcas y país de origen de procedencia de los cigarrillos de tabaco, las mismas que fueron representadas en diagramas de cajas siguiendo las recomendaciones de Boyer, (1997). Finalmente, de realizaron comparaciones entre grupos con el test de Tukey cuyos resultados serán expresados como medias \pm desviación estándar, empleando el software estadístico Minitab versión 17 .

\section{Resultados}

Concentraciones de cadmio en cigarrillos de tabaco de acuerdo a su marca

La concentración promedio de cadmio (Fig. 2) en la población calculada a partir de las 22 muestras de tabaco fue de $1,89 \mathrm{mg} / \mathrm{kg}$. Con un intervalo de confianza del $95 \%$. De manera general, el contenido de Cd varió considerablemente en el tabaco de las marcas comerciales analizadas. El $13.64 \%$ de las concentraciones sobrepasaron los $3 \mathrm{mg} / \mathrm{kg}$. La marca de cigarrillos denominada como COS obtuvo el promedio más alto $(4,73 \pm 1,14 \mathrm{mg} / \mathrm{kg})$, en tanto que en el tabaco de la codificación COU se encontró el menor promedio $(0,42 \pm 0,26 \mathrm{mg} / \mathrm{kg})$, tal como se aprecia en la Tabla 2.

\section{Diferencia de concentraciones de Cd entre las mar-} cas de cigarrillos de tabaco

Existen diferencias significativas en las concentraciones de $\mathrm{Cd}$ entre las diferentes marcas, con un valor $\mathrm{F}=44,40$ y un $\mathrm{p}$-value $<0,00$. Por medio de la prueba de comparaciones en pareja de Tukey (Fig. 3), se establece que existen 3 grupos muy diferentes entre ellos, el primer grupo identificado por la marca COS $(4,73 \pm 1,14 \mathrm{mg} / \mathrm{kg})$, quien posee el mayor promedio, el segundo grupo identificado por la marca NAL $(2,022 \pm 0,23 \mathrm{mg} / \mathrm{kg})$ y un tercer grupo formado por 3 marcas que comparten estadísticamente el mismo promedio, siendo estas NAM, ILS Y COU.

\section{Concentraciones de cadmio en cigarrillos de taba-} co de acuerdo a su país de procedencia

Los cigarrillos procedentes de China presentaron una concentración promedio de 4,73 $\pm 1,14 \mathrm{mg} / \mathrm{kg}$ de $\mathrm{Cd}$, por lo cual esperaríamos encontrar valores que van entre $4,09 \mathrm{mg} / \mathrm{kg}$ y $5,37 \mathrm{mg} / \mathrm{kg}$.

Los cigarrillos de Ecuador poseen una concentración promedio de $1,49 \pm 0,58 \mathrm{mg} / \mathrm{kg}$ de $\mathrm{Cd}$ con valores entre $1,04 \mathrm{mg} / \mathrm{kg}$ y $1,95 \mathrm{mg} / \mathrm{kg}$. Los argentinos poseen una concentración promedio de $0,53 \pm 0,09 \mathrm{mg} / \mathrm{kg}$ con valores entre $-0,49 \mathrm{mg} / \mathrm{kg}$ y 1,54 . Los cigarrillos de la India poseen una concentración promedio $0,42 \pm 0,26$ $\mathrm{mg} / \mathrm{kg}$ con valores entre $-0,23 \mathrm{mg} / \mathrm{kg}$ y $1,06 \mathrm{mg} / \mathrm{kg}$ (Fig. 4, tabla 3). 
Rev. Cient. Cien. Nat. Ambien. 14(1):127-136
Durán \& Castro • Concentración de cadmio en marcas de cigarrillos de tabaco comercializados en Guayaquil, Ecuador

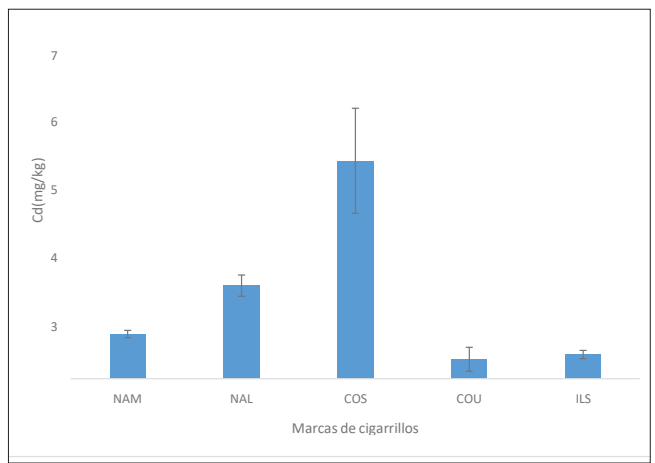

Figura 2. Concentración de Cd en marcas de cigarrillos de tabaco distribuidos en laciudad de Guayaquil; Los resultados se muestran promedios \pm Desviación estándar.

Tabla 2. Resumen de media e intervalo de confianza de Cd de cadmio en cigarrillosde tabaco de según su marca. Los valores están expresados en $\mathrm{mg} / \mathrm{kg}$.

\begin{tabular}{lccc}
\hline Marca & Media & $\begin{array}{r}\text { Desv. } \\
\text { Est. }\end{array}$ & IC de $95 \%$ \\
\hline COS & 4,73 & 1,14 & $(4,19 ; 5,28)$ \\
COU & 0,42 & 0,29 & $(-0,13 ; 0,96)$ \\
ILS & 0,53 & 0,09 & $(-0,34 ; 1,39)$ \\
NAL & 2,02 & 0,23 & $(1,48 ; 2,57)$ \\
NAM & 0,97 & 0,08 & $(0,42 ; 1,51)$
\end{tabular}

\section{Diferencia de concentraciones de $C d$ según su país} de procedencia

Existen diferencias significativas en las concentraciones de $\mathrm{Cd}$ entre los diferentes países de procedencia, con un valor $F=40,08$ y un $p$-value $<0,000$. Por medio de la prueba de comparaciones en pareja de Tukey (Fig. 5), se establece queexisten 3 grupos muy diferentes entre ellos, el primer grupo identificado por China, cuyos cigarrillos poseen la mayor concentración de Cd con un promedio de 4,73 mg/kg, el segundo grupo está formado por Ecuador y Argentina con una concentración promedio de $1,49 \pm 0,58 \mathrm{mg} / \mathrm{kg}$ y $0,525 \pm 0,09 \mathrm{mg} / \mathrm{kg}$, respectivamente. $Y$ un tercer grupo formado por Argentina e India, con una concentración promedio de $0,53 \pm 0,09 \mathrm{mg} / \mathrm{kg}$ e India 0,42 $\pm 0,26$ $\mathrm{mg} / \mathrm{kg}$, respectivamente.

Propuesta para reducir la incidencia de $\mathrm{Cd}$ en el proceso de producción de cigarrillos de tabaco de producción nacional

Realizar análisis de los parámetros más importantes que pueden afectar a la concentración de Cd en suelo

Los parámetros más importantes son: el pH, la deposición atmosférica, el contenido de Cd en fertilizantes fosfatados y en precipitaciones.

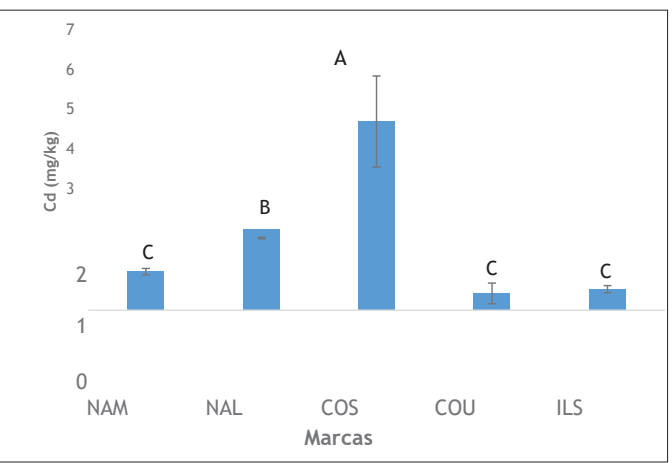

Figura 3. Niveles de cadmio en cigarrillos de tabaco por marca que se comercializanen la ciudad de Guayaquil; letras diferentes indican que hay diferencias significativas según Tukey $(\mathrm{p}<0.05)$. Los resultados están expresados como media \pm desviación estándar.

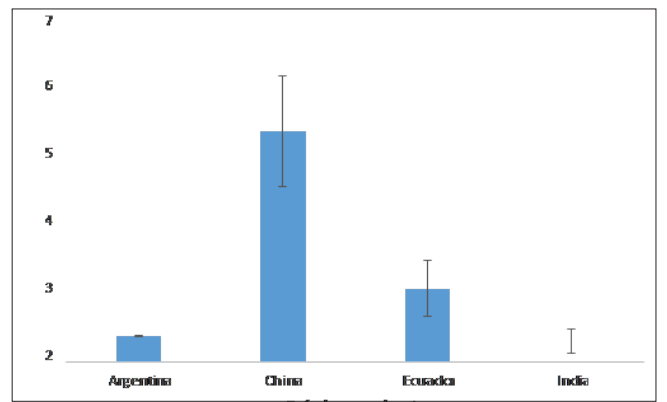

Figura 4. Concentración de $\mathrm{Cd}$ en cigarrillos de tabaco según su país de procedencia; Los resultados están expresados como media \pm Desviación estándar.

Tabla 3. Resumen de media e intervalo de confianza de cadmio en cigarrillos detabaco de según su país de procedencia, los valores están expresados en $\mathrm{mg} / \mathrm{kg}$.

\begin{tabular}{lccc}
\hline País & Media & $\begin{array}{r}\text { Desv. } \\
\text { Est. }\end{array}$ & IC de 95\% \\
\hline ARGENTINA & 0.53 & 0.09 & $(-0.49 ; 1.54)$ \\
CHINA & 4.73 & 1.136 & $(4.09 ; 5.37)$ \\
ECUADOR & 1.49 & 0.578 & $(1.04 ; 1.95)$ \\
INDIA & 0.42 & 0.258 & $(-0.23 ; 1.06)$
\end{tabular}

Estos parámetros deberán medirse y reportarse antes de trasladar las plantas del semillero hacia su siguiente etapa en el campo.

Medios de control: Reportes, informes, auditorías.

Capacitar a los agricultores acerca de las medidas in situ para disminuir las concentraciones inciales de Cd en suelo Según Wagner (1993) los factores más importantes que regulan la absorción de $\mathrm{Cd}$ son las 
Rev. Cient. Cien. Nat. Ambien. 14(1):127-136 Junio 2020
Durán \& Castro • Concentración de cadmio en marcas de cigarrillos de tabaco comercializados en Guayaquil, Ecuador

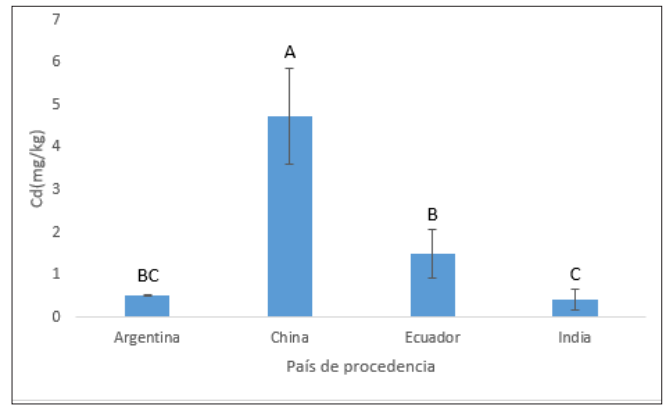

Figura 5. Niveles de cadmio en cigarrillos de tabaco que se comercializan en la ciudad de Guayaquil según país de procedencia; letras diferentes indican que hay diferencias significativas según Tukey $(\mathrm{p}<0.05)$. Los resultados están expresadoscomo media \pm desviación estándar.

concentraciones iniciales de $\mathrm{Cd}$ presente en el suelo y el $\mathrm{pH}$, se proponen las siguientes medidas:

Se propone utilizar las plantas de tabaco que quedaron fuera de la selección para su cultivo, aprovechando su característica hiperacumuladora para reducir los niveles de $\mathrm{Cd}$ presentes en el suelo a cultivar.

Puesto que la solubilidad y por consiguiente biodisponibilidad de los metales pesados es mayor cuando el $\mathrm{pH}$ es ácido, se propone realizar correcciones de suelos ácidos (enmiendas calizas o encalado). Se sugiere utilizar cal agrícola (caliza), debido a su bajo coste si comparamos con otras enmiendas y porque su efecto es el más duradero en el suelo. Dicha enmienda es la más utilizada para suelos de uso agrícola.

Medios de control: Acta de reuniones de capacitación, supervisión en el campo.

1. Restringir el uso de fertilizantes y pesticidas con niveles elevados demetales pesados

Investigadores como Alloway y Steinnes (1999) concuerdan en que el factor antropogénico más importante de la contaminación por Cd son los "depósitos directos"; es decir, el uso de fertilizantes fosfatados, por ende, si prohibimos su uso se reduciráde manera importante la incidencia de $\mathrm{Cd}$ en el producto final.

Una alternativa al uso de estos fertilizantes fosfatados que ha dado buenos resultados es la aplicación de cenizas de lodos, se puede explicar mediante informesque defienden que el fósforo en cenizas de lodos de aguas residuales puede ser másaccesible por las plantas, especialmente en suelos más ácidos (Rodríguez, 2015).

Medios de control: normativas, políticas internas, reportes, informes, auditorías.

\section{Implementar controles más exhaustivos acerca de los aditivos químicosempleados en el proceso.}

En primer lugar, se debería sincerar todos los aditivos químicos que se empleenen el proceso, actualmente estos no se encuentran enlistados en la información acerca del proceso de producción de tabaco que se encuentra disponible en el sitio web oficialde Philip Morris International. Posterior a esto, la empresa debería elaborar informes de toxicidad de cada producto químico utilizado y con base en eso tomar las decisionesacerca del aditivo químico a utilizar.

Medios de control: Disponibilidad de información, informes, reportes.

\section{Discusión}

Independientemente de la marca y su país de procedencia, todas las muestras de tabaco presentaron niveles de $\mathrm{Cd}$, siendo la marca $\mathrm{COS}$ de origen chino la que obtuvo las concentraciones más elevadas $(5,92$ $\mathrm{mg} / \mathrm{kg}$ ). El nivel promedio de $\mathrm{Cd}$ en las 22 muestras de cigarrillos de las marcas analizadas que se expenden en Guayaquil es de 1,89 $\pm 1,758 \mathrm{mg} / \mathrm{kg}$. Las variaciones en las concentraciones tóxicas demetales pesados en los cigarrillos de tabaco pueden depender además de los niveles de Cd en los hábitats del suelo, del uso de fertilizantes y tratamientos con pesticidas, además de las condiciones de crecimiento (Cabrera et al., 1995) y la contaminación industrial (Saarenen et al., 1989).

Watanabe et al. (1987), reportó en cigarrillos de origen chino resultados con unamedia de $0,93 \mathrm{mg} / \mathrm{kg}$, mientras que en el presente estudio el promedio de concentraciones de $\mathrm{Cd}$ fue 5 veces mayor, lo cual es preocupante debido evidencia el incremento de la presencia de este metal en suelos agrícolas probablemente ubicadosdonde también se cultivan productos utilizados en la alimentación humana. Otros datos más recientes, reportados por O'Connor et al. (2009) también indican que los cigarrillos de tabaco de varias marcas producidas en China presentan concentraciones similares $(3,21 \mathrm{mg} \mathrm{Cd} / \mathrm{kg})$, en comparación con los resultados que se presentan en este estudio. Sin embargo, por la evidencia obtenida no se debe asumir que todos los cigarrillos de procedencia China sean los más contaminados con $\mathrm{Cd}$, ya que siendo este país el principal productor de cigarrillos en el mundo con una extensión destinada para la agricultura de 5'277,330 km (Worldbank, 2019) posee muchos tipos de cultivares, cada uno con características distintas.

Cabe destacar que los cigarrillos procedentes de la India, aunque también ingresan de manera ilícita presentaron los menores niveles de Cd, también perjudicanal estado, pero esta vez de manera tributaria, ya que siendo cigarrillos de mejor calidad(refiriéndose únicamente a niveles de $\mathrm{Cd}$ ) deberían pagar mayores impuestos.

En estudios realizados al tabaco procedente de India, Watanabe et al. (1987) se reportó una concentración promedio de $0,35 \mathrm{mg} / \mathrm{kg}$, similares a los hallados en este estudio $(0,41 \pm 0,26 \mathrm{mg} / \mathrm{kg})$. Estos datos sugieren que los suelos agrícolas de la India presentan bajas concentraciones iniciales de $\mathrm{Cd}$, debido entre otros 
Rev. Cient. Cien. Nat. Ambien. 14(1):127-136 Junio 2020
ISSN: $2773-7772$
Durán \& Castro • Concentración de cadmio en marcas de cigarrillos de tabaco comercializados en Guayaquil, Ecuador factores a leyes y políticas agroecológicas estrictas. Esto último, se respalda con el caso de Sikkim; estado agrícola de India que se convirtió en el primer estado orgánico del mundo, lo cual lo hizo acreedor del premio de oro en el Future Policy Award (FPA) celebrado en 2018 (FAO, 2018).

Por otra parte, autores como Lugon et al. (2006) quienes analizaron las concentraciones promedio de $\mathrm{Cd}$ en cigarrillos de tabaco provenientes de 13 países, entre estos Ecuador, reportaron para dicho país, una concentración promedio de $1,95 \mathrm{mg} / \mathrm{kg}$. Este resultado es similar al del presente estudio, donde se obtuvo una media de $1,495 \mathrm{mg} / \mathrm{kg}$. Dado a que existen 14 años de diferencia entre ambos estudios, se podría asumir que los niveles de Cd en el tabaco de los cigarrillos manufacturados en el Ecuador han experimentado una aparente disminución, sin embargo, se requiere un estudio más profundo para conocer si este resultado es significativo.

Algo que llama la atención en las concentraciones promedio de Cd en las dos marcas de cigarrillos de tabaco de origen ecuatoriano, es que pese a ser producidas por la misma compañía reportaron concentraciones diferentes. Esta diferencia evidencia que los suelos agrícolas de donde provienen las hojas de tabaco para cadamarca tienen características diferentes; debido a que los cultivares se encuentran ubicados principalmente en las provincias de Guayas y Los Ríos.

Dado que la mayor fuente de contaminación por Cd se debe las condiciones iniciales del suelo (Wagner, 1993), es conveniente realizar análisis de Cd en el mismoprevio a su uso agrícola. En Ecuador, se ha demostrado la presencia de $\mathrm{Cd}$ en suelosagrícolas donde se cultiva cacao en las provincias de Guayas y El Oro (Chávez et al., 2015), a distintas profundidades, se obtuvieron medias de 1,54 mg/kg $(0-5 \mathrm{~cm}), 1,39 \mathrm{mg} /$ $\mathrm{kg}(5-15 \mathrm{~cm}), 0,77 \mathrm{mg} / \mathrm{kg}(15-30 \mathrm{~cm}), 0,85 \mathrm{mg} / \mathrm{kg}$ $(30-50 \mathrm{~cm})$. Esto nos indica la probabilidad de que los suelos agrícolas de la provincia del Guayas donde se cultivael tabaco nacional presenten contaminación por Cd.

En el tabaco proveniente de Argentina se han reportado concentraciones, promedio de $0,65 \mathrm{mg} / \mathrm{kg}$ de $\mathrm{Cd}$ (Lugon et al., 2006), superando a la obtenida en el presente estudio $(0,53 \pm 0,09 \mathrm{mg} / \mathrm{kg})$ con la marca argentina importada legalmente (ILS), evidenciando en ambos casos una baja concentración de Cd. Estos resultados no presentan mucha variación, lo cual podría deberse a que las concentraciones iniciales de $\mathrm{Cd}$ en sus cultivos son bajas.

Curiosamente, las medias de los niveles de Cd, tanto las más altas como las más bajas, se reportaron en las muestras de tabaco más económicas y de comercio ilícito correspondientes a los países de China e India, respectivamente. De igual manera ambas marcas gozan de una gran popularidad que podría atribuirse a su PVP.

Los resultados del presente trabajo indican que existe una amplia diferencia entre concentraciones de $\mathrm{Cd}$ de cigarrillos de tabaco producidos en Ecuador frente a los que entran de manera ilícita, este resultado se encuentra en desacuerdo con los de Petit de Peña et al. (2014), quienes reportaron que no existe diferencia significativa entre el $\mathrm{Cd}$ detectado en cigarrillos de tabaco producidos en Venezuela versus los cigarrillos que entran a dicho país por contrabando, a diferencia de este estudio el tabaco ilegal que ingresa al mercado venezolano son de diferente origen.

Es evidente que las plantas de tabaco cultivadas en ambientes de suelocontaminados acumulan esos metales pesados en sus hojas. De hecho, los cultivaresde tabaco han sido reportados como grandes acumuladores de metales, especialmente metales tóxicos (Miner et al., 1997; Pinto \& Ferreira, 2015). La acumulación de metales pesados tóxicos en el cultivo de tabaco dependen de los órganos de las plantas, con la peculiaridad de que la planta de tabaco acumula mayor concentración de metales pesados en sus hojas a diferencia de la mayoría de las plantas cuyo órgano acumulador suele ser las raíces en primer lugar (Adamu et al., 1989). En este caso, dicha peculiaridad resulta negativa al ser las hojas de la planta de tabaco el órgano usado para la elaboración de los cigarrillos.

Por otro lado, estadísticas muestran cómo el consumo de tabaco ha disminuidoen los últimos años, tanto en países desarrollados como en vías de desarrollo (Ritchie\& Roser, 2020). Sin embargo, estos datos no deberían considerarse reales debido a que no consideran la data de los cigarrillos que entran de manera ilícita a países comoEcuador. Se especula que la población fumadora con bajo poder adquisitivo se inclinahacia estas marcas al momento de comprarlas, por su bajo precio y mayor contenido de cigarrillos por cajetilla, volviéndolos los más populares y por consiguiente exponiendo a la población fumadora a cigarrillos de tabaco con mayores niveles de $\mathrm{Cd}$.

Ahora bien, tomando en cuenta los resultados de la encuesta realizada por el INEC en el año 2017, la que indica que en Guayaquil se consumen en promedio 49 cigarrillos semanales concluimos que la población fumadora promedio y más aún las personas que consumen el cigarrillo COS se encuentran en peligro debido a las elevadas concentraciones de $\mathrm{Cd}$ que contienen los cigarrillos comercializados en la ciudadde Guayaquil. La población fumadora está comprometiendo su salud y se enfrentan a padecer enfermedades asociadas al Cd, como cánceres de pulmón, de esófago, de boca, garganta, laringe, riñón, entre otros, así como también enfermedades delcorazón y apoplejía (U.S. Department of Health and Human Services, 2010).

Cabe señalar que no hay datos suficientes sobre las concentraciones de cadmioen las marcas de cigarrillos comercializadas en Ecuador por parte de organismos estatales de regulación y control, y tampoco por parte de los propios fabricantes. Esteestudio proporciona nuevos datos que pueden ser de utilidad para las autoridades sanitarias nacionales e internacionales. Además, los resultados obtenidos brindan información 
Rev. Cient. Cien. Nat. Ambien. 14(1):127-136 Junio 2020
Durán \& Castro • Concentración de cadmio en marcas de cigarrillos de tabaco comercializados en Guayaquil, Ecuador muy importante para el conocimiento de la población fumadora delEcuador.

De igual manera, los resultados presentados en este estudio se pueden considerar como un argumento más para el no consumo de cigarrillos de tabaco, debido a que demuestra el contenido de un contaminante altamente tóxico incluso a bajas concentraciones como el $\mathrm{Cd}$ y su repercusión en la salud humana.

\section{Conclusiones}

Los análisis realizados comprobaron la presencia de cadmio en los cigarrillos de tabaco de producción nacional e internacional comercializados en la ciudad de Guayaquil., Existiendo diferencias significativas entre los cigarrillos producidos en el país $(1,49 \pm 0,578)$ frente a los que ingresan de manera ilícita $(4,73 \pm 1,14 \mathrm{mg} / \mathrm{kg})$.

Los cigarrillos provenientes del comercio ilícito, cuya procedencia es de China $(4,73 \pm 1,14 \mathrm{mg} / \mathrm{kg})$, presentaron las concentraciones más altas, mientras que los cigarrillos provenientes del comercio ilícito cuya procedencia fue de India presentaronpromedios de concentraciones de $\mathrm{Cd}$ bajas $(0,42 \pm 0,26 \mathrm{mg} / \mathrm{kg})$.

La marca proveniente de Argentina (ILS) de tabaco listo para enrolar, a pesar de ser la más costosa y exclusiva no fue la que menor concentraciones de Cd presentó, compartiendo casi las mismas características con los cigarrillos de tabaco producidosen Ecuador.

Los cigarrillos de producción nacional $(1,49 \pm 0,58 \mathrm{mg} /$ $\mathrm{kg}$ ) ocupan el segundo lugar en lo que respecta a mayores niveles de $\mathrm{Cd}$. Además, existen diferencias significativas entre las 2 marcas de cigarrillos de producción nacional, identificadas porNAL y NAM, (por cuestiones éticas no podemos indicar los nombres comerciales de las mismas), aun perteneciendo a la misma compañía.

No existen normativas acerca de los límites máximos permisibles para $\mathrm{Cd}$ en cigarrillos de tabaco, ni en Ecuador ni en ningún otro país.

Finalmente, se determina la concentración de Cd en los cigarrillos de tabaco que son comercializados en la ciudad de Guayaquil difiere en función a la marca y país de origen.

\section{Recomendaciones}

- En vista de la incidencia y toxicidad del Cd en los cigarrillos analizados en el presente estudio se recomienda la creación de límites máximos permisibles para $\mathrm{Cd}$ en cigarrillos de tabaco.

- Se recomienda analizar al proveedor de la marca de cigarrillos chinos cuyas concentraciones de $\mathrm{Cd}$ fueron extremadamente elevadas, comparándolos con otros cigarrillos de tabaco de la misma procedencia, pero con distintos proveedores y marcas.

- Se recomienda a los organismos de regulación y control que tomen acción para detener el ingreso al país de productos de contrabando.
- A partir del presente estudio, se debería realizar una campaña publicitaria que concientice a la población fumadora de los peligros que corren al exponerse a una fuente tan importante de $\mathrm{Cd}$ como lo es el cigarrillo de tabaco, así como la gran diferencia de concentraciones de dicho contaminante detectadas en una marca de cigarrillos de contrabando.

\section{Bibliografía}

Adamu, C. A., Bell, P. F., Mulchi, C., \& Chaney, R. 1989. Residual metal concentrations in soils and leaf accumulations in tobacco a decade following farmland application of municipal sludge. Environmental Pollution, 56(2): 113- 126.

Alloway, B. J., y Steinnes, E. 1999. Anthropogenic Additions of Cadmium to Soils.

Cadmium in Soils and Plants, 97-123. Springer Dordrecht. doi:10.1007/978-94-011-4473-5_5

Ashraf, M.A., Maah, M. J. y Yusoff, I. 2014. Soil Contamination, Risk Assessment and Remediation. Environmental Risk Assessment of Soil Contamination 1:3-56. doi: $10.5772 / 57287$

Boyer, J., J. Fouqurean, y R. Jones. 1997. Spatial characterization of water quality in Florida Bay and Whitewater Bay by multivariate analyses: Zones of similar influence. Estuaries, 20(4): 743-758.

Cabrera, C., Lorenzo, M. L., y Lopez, M. C. 1995. Lead and cadmium contamination in dairy products and its repercussion on total dietary intake. Journal of Agricultural and Food Chemistry, 43(6): 1605-1609.

Cai, J., Liu, B., Zhu, X., y Su, Q. 2002. Determination of pyrethroid residues in tobacco and cigarette smoke by capillary gas chromatography. Journal of Chromatography A, 964(1-2): 205-211.

Chakraborty, S., Dutta, A., Sural, S., Gupta, D., y Sen, S. 2013. Ailing bones and failing kidneys: a case of chronic cadmium toxicity. Annals of Clinical Biochemistry, 50 (5): 492-495.

Chavez, E., He, Z., Stoffella, P., Mylavarapu, R., Li, Y., Moyano, B., y Baligar, V. 2015. Concentration of cadmium in cacao beans and its relationship with soil cadmium in southern Ecuador. Science of the Total Environment, 533: 205-214.

Combariza, D. 2009. Contaminación por metales pesados en el embalse de Muña y su relación con los niveles en sangre de plomo, mercurio y cadmio y alteraciones de salud en los habitantes del municipio de Sibaté (Cundinamarca). Tesis de maestría. Universidad Nacional de Colombia. Bogotá.

Davis, R. D. 1984. Cadmium - A complex environmental problem Part II. Experientia,40(2): 117-126.

FAO. 2018. El "Estado 100\% orgánico" Sikkim en India gana el oro. Políticas de Brasil, Dinamarca y Ecuador galardonadas con la plata. Recuperado 28 enero, 2020, de http:// www. fao.org/agroecology/slideshow/news-article/ es/c/1157017/

Flores, E., Pozo, W., Pernía, B., y Sánchez, W. 2018. Niveles de cadmio en atún fresco y enlatado para consumo humano en Ecuador. Maskana, 9(2): 35-40.

Herrera, T. 2000. La contaminación con cadmio en suelos agrícolas. Venesuelos, $8(1,2)$ : 42-47.

Instituto de Salud Pública de Chile. 2019. Sección Química Ambiental: Contaminación Ambiental. Recuperado de http://www.ispch.cl/saludambiental/ambiente/ quimica_ambiental/contaminacion

Leshner, Al. 2001. Nicotine Addiction (No. 01-4342). Research Report Series. National Institute on Drug Abuse.

Londoño, L., Londoño, P., y Muñoz, F. 2016. Los riesgos de los 
Rev. Cient. Cien. Nat. Ambien. 14(1):127-136

Junio 2020
Durán \& Castro • Concentración de cadmio en marcas de cigarrillos de tabaco comercializados en Guayaquil, Ecuador metales pesados en la salud humana y animal. Biotecnología en el Sector Agropecuario y Agroindustrial, 14(2): 145-153.

López, J.D. 2017. Determinación de cadmio y plomo en soya (Glycine max L.) en grano,en polvo y líquida comercializada en la ciudad de Guayaquil Ecuador. Tesis de grado previa a la obtención de título de Ingeniero Ambiental. Universidad de Guayaquil.

Lugon-Moulin, N., Martin, F., Krauss, M. R., Ramey, P. B., y Rossi, L. 2006. Cadmium concentration in tobacco (Nicotiana tabacum L.) from different countries and its relationship with other elements. Chemosphere, 63(7): 1074-1086.

Miner, G. S., Gutierrez, R., y King, L. D. 1997. Soil factors affecting plant concentrations of cadmium, copper, and zinc on sludge-amended soils. Journal of Environmental Quality, 26(4): 989-994.

Ministerio de Salud Pública. 2018. Ministerio de Salud garantiza acceso a lasalud de pacientes con cáncer. Recuperado de https://www.salud. gob.ec/ministerio-de-salud-garantiza-acceso-a-la-saluddepacientes-con-cancer-2/

Muñoz, J. 2017. Determinación de Cadmio en fertilizantes, plantas de Oryza sativa L. y suelos de la provincia del Guayas: Propuesta de saneamiento. Tesis de grado previa a la obtención de título de Ingeniero Ambiental. Universidad de Guayaquil.

Nava, C. \& Méndez, M. 2011. Efectos neurotóxicos de metales pesados (cadmio, plomo, arsénico y talio). Archivos de Neurociencias 16(3): 140-147.

O'Connor, R. J., Li, Q., Stephens, W.E., Hammond, D., EltonMarshall, T., Cummings,

K.M., y Fong, G.T. 2010. Cigarettes sold in China: design, emissions and metals. Tobacco Control, 19(Supplement 2), i47-i53.

Okrent D. 1999. On intergenerational equity and its clash with intragenerational equity and on the need for policies to guide the regulation of disposal of wastes and other activities posing very long-term risks. Risk Analysis, 19(5): 877-901.

Pernía, B., Mero, M., Muñoz, J., Bravo, K., Morán, N., Zambrano, J., y Cornejo, X. 2016. Plantas acuáticas con potencial para fitoextracción de Cadmio enarrozales del Cantón Daule, provincia del Guayas, Ecuador. Revista Científica de Ciencias Naturales y Ambientales, 10(2): 37-51.

Petit de Peña, Y., Guillén, J., Vicuña, N., Briceño, L., Carrero P., yPeñaloza, H. 2014.Determinación del contenido de cadmio en muestras de tabaco de cigarrilloscomercializados en Venezuela. Revista de la Facultad de Farmacia 56(1): Jan. 2014, pp. 26+. Gale OneFile: Informe Académico, link.gale.com/apps/doc/A466166097/ IFME? $\mathrm{u}=$ anon $1855546 \mathrm{~d} \&$ sid=googleScholar\&xid=b3b3f2a9. Accessed 7 Oct. 2021.

Pinto, E. y Ferreira, I. 2015. Cation transporters/channels in plants: tools for nutrientbiofortification. Journal of Plant Physiology, 179: 64-82.

Ramírez, A. 2002. Toxicología del cadmio. Conceptos actuales para evaluar exposición ambiental u ocupacional con indicadores biológicos. Anales de la Facultad de Medicina. 63: 51-64.

Reyes, Y., Vergara, I., Torres, O., Díaz, M., y González, E. 2016. Contaminación por metales pesados: Implicaciones en salud, ambiente y seguridad alimentaria. Revista Ingeniería, Investigación y Desarrollo, 16(2): 66-77.

Ritchie, H. y Roser, M. 2020. Smoking. Published online at Our World in Data. Retrievedfrom: https: / /ourworldindata.org/ smoking [Online Resource]

Rodríguez, C. 2015. Effects of alternative phosphorus fertilizers on phosphorusmobilization b y different cover crops in two soils. Memoria, Department for Sustainable Agricultural Systems, Division of Organic Farming. Universität für Bodenkulture Wien.

Saarenen, M., Kantola, M., Saarikoski, S., y Vanha-Perttula, T. 1989. Human Seminal Plasma Cadmium: Comparison with Fertility and Smoking Habits/Cadmium in menschlichem Seminalplasma: Vergleich zwischen Fertilität und Rauchgewohnheiten. Andrologia, 21(2): 140-145.

Sardar, K., Ali, S., Hameed, S., Afzal, S., Fatima, S., Shakoor, M., Bharwana, S., y Tauqeer, H. 2013. Heavy Metals Contamination and what are the Impacts on Living Organisms. Greener Journal of Environmental Management and Public Safety, 2(4): 172-179.

Singh, P., y Tewari, R. 2003. Cadmium toxicity induced changes in plant water relations and oxidative metabolism of Brassica juncea L. plants. Journal of EnvironmentalBiology, 24: 107-112.

Smith, J., Livingston, D., y Doolittle, D. 1997. An international literature survey of "IARCGroup I carcinogens" reported in mainstream cigarette smoke. Food. Chem. Toxicol. 35(1011): 1107-1130.

U.S. Department of Health and Human Services. 2010. How Tobacco Smoke CausesDisease: The Biology and Behavioral Basis for Smoking-Attributable Disease: A Report of the Surgeon General. Atlanta, GA: U.S. Department of Health and Human Services, Centers for Disease Control and Prevention, National Center for Chronic Disease Prevention and Health Promotion, Office on Smoking and Health, 2010. Available from: https://www.ncbi.nlm.nih.gov/books/ NBK53017/

U.S. Environmental Protection Agency. 2007. Method 7010. Graphite Furnace AtomicAbsorption Spectrophotometry. Retrieved from https://www.epa. gov/sites/production/files/2015-12/documents/7010.pdf

Wagner, G.J. y Yeargan, R. 1986. Variation in Cadmium Accumulation Potential and Tissue Distribution of Cadmium in Tobacco. Plant Physiology, 82(1): 274-279.

Wagner, G.J. 1993. Accumulation of cadmium in crop plants and its consequences to human health, Advances in Agronomy, 51: 173-212.

Watanabe, T., Kasahara, M., Nakatsuka, H., y lkeda, M. 1987. Cadmium and lead contents of cigarettes produced in various areas of the world1. The Science of The Total Environment, 66: 29-37.

World Bank. 2019. World Development Indicators [Conjunto de datos]. Recuperado

7 febrero, 2020, de https://data.worldbank.org/ indicator/AG.LND.ARBL.ZS?view=chart

Yoshihara, T., Suzui, N., Ishii, S., Kitazaki, M., Yamazaki, H., Kitazaki, K., y Fujimaki,

S. 2013. A kinetic analysis of cadmium accumulation in a Cd hyper- accumulator fern Athyrium yokoscense and tobacco plants. Plant, Cell \& Environment, 37(5): 1086-1096. 